\title{
Study on the Ultrasonic Levitation Supporting Technique of Motor Rotor
}

\author{
Wang Hongchen ${ }^{1,2}$ and Yang Zhigang ${ }^{1}$ \\ ${ }^{1}$ School of Mechanical Science and Engineering of Jilin University, Changchun, \\ Jilin, 130025 \\ ${ }^{2}$ Engineering Training Center of Changchun Institute of Technology, Changchun, \\ Jilin, 130012 \\ whcwanghongchen@126.com,yzg@jlu.edu.cn
}

\begin{abstract}
This essay presents the theory about the levitation support of the ultrasonic vibration for the motor rotor, designs an ultrasonic levitation support structure, and theoretically calculates the levitation force by adopting the conical radiation surface to provide the levitation support for the motor rotor. By using the designed device, this essay analyzes and tests the bearing capacity, obtains the relationship between the levitation force and the levitation clearance, and conducts the experimental studies to the relationship between the highest speed of the rotor and the levitation clearance. The results show that the ultrasonic vibration can enhance the supporting effect and increase the maximum speed of the rotor by reducing the clearance, therefore achieve the levitation of the rotor.
\end{abstract}

Keywords: Suspension technology, Rotor, Suspension clearance, Levitation force, Speed

\section{Introduction}

High speed motor usually refers to the motor that the speed exceeds $10000 \mathrm{r} / \mathrm{min}$. It is widely used in industries, for example, high speed machine tool, the turbo molecular pump, high speed centrifuge, precision gyroscope, flywheel energy storage, and so on. It shows the increasingly important and broad application prospects. The rotor of the traditional high speed motor is supported by two mechanical bearings, which has great friction and wear, low life, poor reliability, and produces serious mechanical vibration and noise. This has been unable to meet the supporting requirements of high speed motor. Current methods to solve this problem are to use the non-contact air, liquid or magnetic bearing, which can basically achieve the low friction or friction free operation. However, it also brings some problems, such as the complex structure of the system, high energy consumption, low efficiency, large volume, and the complex control system. These problems seriously restrict the further development and application of high speed motor [1-4]. In recent years, some people proposed the bearingless motor technology. The current research work is mainly focused on experimental research, technology development, and proving the feasibility of the application of motors with various structures in the bearingless motor technology. It is still in the stage of exploration and research for international studies of the bearingless motor. In the process of the research on the surface friction properties of the ultrasonic vibration object, it shows that the surface of the ultrasonic vibration object can reduce the friction factor of the contact surface, and if the amplitude is large enough, it can suspend the object for a certain distance.

Based on this, it is proposed in this essay to solve the problem of the levitation support of motor rotor by using the ultrasonic levitation supporting technique. This essay studies the basic theory and the key technological problems in the process of this support, tests 
the levitation support of motor rotor via experimental devices and the stability of high speed rotation, thus forms a new technique or method for the structure of high speed motor, and then lays the foundation of developing a new type of high speed motor with more practical value.

\section{Ultrasonic Levitation Technology}

Ultrasonic levitation technology was first found by Kundt in 1886. Professor Ueha Sadayuki in Tokyo Institute of Technology has proved that the dynamic surface pressure of the flat is up to $0.75 \mathrm{Mpa}$ [5-6] when it vibrates in the frequency of $40 \mathrm{kHz}$ and in the amplitude of $2 \mu \mathrm{m}$; Takaaki Oiwa has applied the ultrasonic levitation technology to the disk rotor bearing, and developed the ultrasonic levitation system that can limit the axial and radial motion of the rotor to an approximate speed of 120rpm under the effect of inertia [7]; Tadeusz and colleagues have designed the radial ultrasonic levitation supporting structure, which can provide the pneumatic drive to the shaft by letting high frequency vibration transmitting to the inner surface of cylinder through flexure hinge mechanism, and the speed can reach $1020 \mathrm{rpm}$ [8]; intensive studies on the relationship between ultrasonic amplitude and friction factor have been conducted by Piezoelectric drive and Control Technology Laboratory of Jilin University[9-21], the results show that:

(1) When the ultrasonic vibration amplitude is less than the sum of the surface roughness $\mathrm{Ra}$ of two contact surfaces, it is performed as the friction reduction effect. It means to decrease the friction factor of two surfaces can reduce the friction factor to the $10 \%$ of the static friction state, so that greatly reduce the frictional wear between the contact surfaces. (2) When the ultrasonic vibration amplitude is more than the sum of the surface roughness Ra of two contact surfaces, it is performed as the suspension effect. It means the larger the amplitude the greater the clearance. When the ultrasonic vibration amplitude is large enough, it can form the absolute suspension on the object.



Figure 1. Curves for the Relationship between Amplitude and Friction Factor

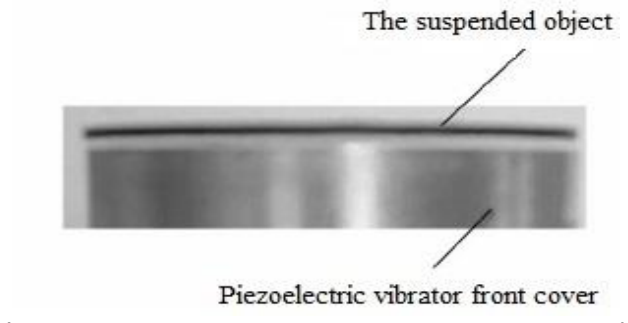

(Weight $30 \mathrm{~g}$, levitation clearance $1.5 \mathrm{~mm}$ )

Figure 2. Schematic Diagram of Ultrasonic Levitation Effect

As long as there is a certain levitation force, it can form the levitation support. The research results show that the density of the levitation force formed by the near-field 
acoustic levitation (the levitation clearance is dozens of microns) can reach about $8.5 \mathrm{~N} / \mathrm{cm}^{2}$ [22]. It indicates the ultrasonic levitation technology has the research value and wide prospect in the application of the high-speed motor spindle levitation.

\subsection{Ultrasonic Levitation Supporting Structure}

The ultrasonic levitation supporting structure established in this essay as shown in Figure 3 is mainly formed by the piezoelectric transducer (langevin transducer), the motor shaft (rotor), the stator coil and the shell, the transducer bracket and the stator frame, etc. To modify the permanent magnet synchronous motor spindle, demolishes the original ball bearing and installs the conical structure at both ends of the motor shaft so that the inner surface matches the outer conical surface at the front-end of the piezoelectric transducer. The axis direction of the motor shaft is perpendicular to the horizontal plane. The piezoelectric transducers at both ends produce the first-order longitudinal vibration under the excitation of the ultrasonic frequency $\mathrm{AC}$ voltage, which forms the levitation clearance between the inner and outer conical surfaces and provides the ultrasonic levitation support to the motor rotor. The stator casing is fixed on the bracket, inside of which the tree-phase stator windings distribute in the stator inner circle according to certain rules. When the power is on, the rotating magnetic field will be formed in the air gap between $\mathrm{p}-\mathrm{m}$ rotor and stator, and drive the motor shaft with permanent magnet to rotate.

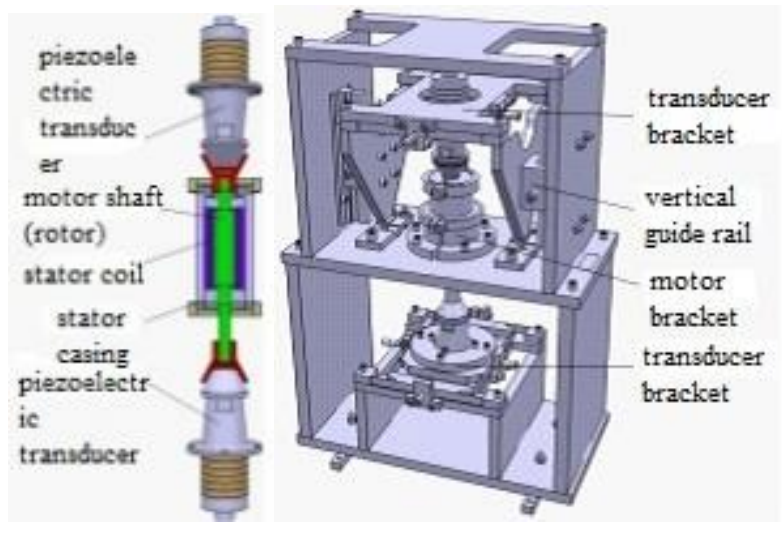

Figure 3. Schematic Diagram of Ultrasonic Levitation Supporting Structure

\section{The Levitation Force of the Cone Type Ultrasonic Levitation Support}

Figure 4 is the Schematic diagram of ultrasonic levitation theory. In the diagram, $f$ is the resonant frequency of piezoelectric ultrasonic transducer, $\alpha$ is the taper angle of the transmitting terminal on the transducer, $G$ is the force of gravity on the rotor, $F_{1}, F_{2}, F_{3}, F_{4}$ are the radiation pressure of the transmitting terminal of the transducer along the conical surface and the axial direction. Because of the force of gravity on the rotor, the levitation clearance $h$ between the rotor and the emitting ends of the transducers A and B is not equal, $h_{1}<h_{2}$. According to the Equation (4), the resultant $F$ of the ultrasonic to the axial radiation pressure of the rotor is in the vertical direction, contrary to the direction of gravity, and the radial radiation pressure is in the circular distribution, therefore, the resultant is 0 . 


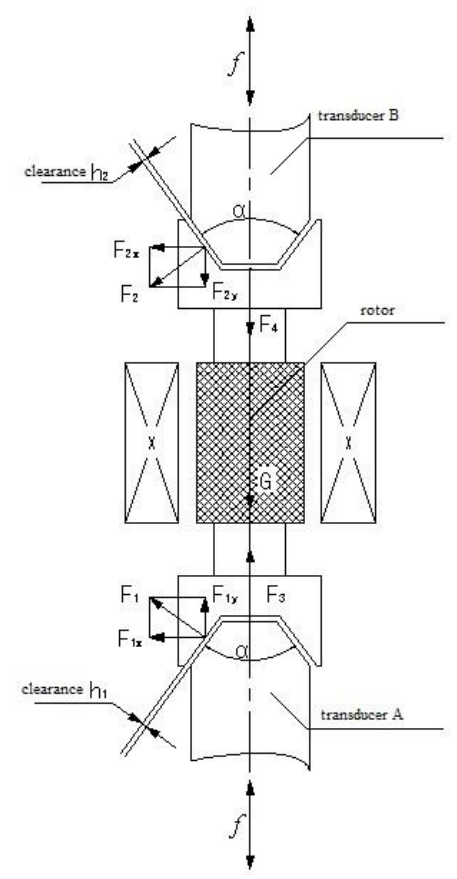

Figure 4. Schematic Diagram of Cone Type Ultrasonic Levitation Support

$\Delta S_{i}$ as the unit area under the effect of the radiation pressure, the force of the radiation pressure on the rotor is:

$$
F=\sum\left(p_{\text {radia }, i} \times \Delta S_{i}\right)
$$

The axial force on the rotor as follows:

$$
\begin{aligned}
& F_{1 y}=F_{1} \cdot \sin \frac{\alpha}{2} \\
& F_{2 y}=F_{2} \cdot \sin \frac{\alpha}{2} \\
& F_{y}=F_{3}+F_{1 y}-F_{4}-F_{2 y}
\end{aligned}
$$

When $F_{y}=G$, the rotor is in the status of the axial force balance, and this is the principle of ultrasonic levitation support for motor rotor.

\section{Test to the Clearance of the Shafting Ultrasonic Levitation Support}

In the condition of closed support, it is not easy to measure the clearance volume of the rotor. We have designed a testing device as shown in Figure 5, in order to test the levitation clearance between the two contact surfaces in ultrasonic vibration environment. It adopts a single piezoelectric transducer supporting shaft, and the taper angles of the conical structures are equal for the piezoelectric transducer radiation surface and the rotor. In the process of testing, the whole test system is placed on the anti-vibration table, to avoid the adverse effects caused by external vibration and to reduce the measurement error. When piezoelectric transducer is not in vibration, we record the reading $h_{1}$ of the laser micrometer, then apply the high frequency sine signal to piezoelectric transducer, keep it in the resonant state and suspend the shaft for a certain clearance. After it is stable, we record the reading $h_{2}$ of the micrometer. The difference between the two readings is the levitation clearance, i.e., $\Delta h=h_{2}-h_{1}$. By the following equation, we calculate the 
levitation clearance $h$ :

$$
h=\Delta h \cdot \sin \frac{\theta}{2}
$$

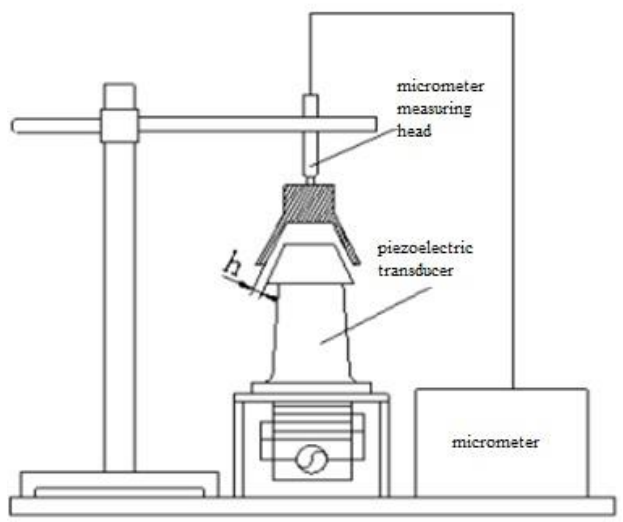

Figure 5. The Measurement of Axial Bearing Capacity

Relationship between levitation force and the clearance is shown in Figure 6. The experimentally measured values and the calculated curves comparatively coincide. When the transducer is in the resonant state, the levitation clearance decreases; meanwhile, the axial levitation bearing capacity increases significantly.

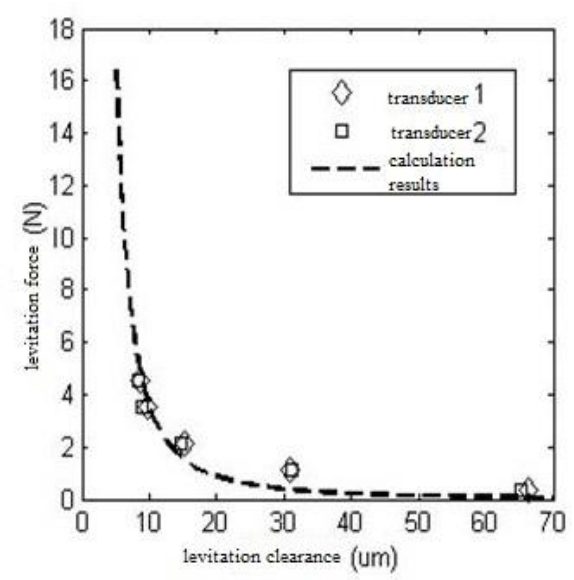

Figure 6. The Relationship between the Axial Levitation Force and the Levitation Clearance

Considering the variations of the clearance and the pressure are small, the first-order trace of the pressure change can be written as:

$$
p=A \cos 2 \pi f t+B \sin 2 \pi f t
$$

$A \cos 2 \pi f t$ changes synchronously with the vibration velocity, known as the damping force; $B \sin 2 \pi f$ changes synchronously with the vibration displacement, known as the stiffness force. By conducting the harmonic response analysis on the conical squeeze film model, we obtain the distribution of the pressure changes $p_{I m}$ caused by the vibration displacement. According to the following equation, we calculate the axial and radial stiffness coefficients $K_{z}, K_{r}$, and the relationship between the stiffness coefficient and levitation clearance is shown in Figure 7. 


$$
K_{z}=\frac{\iint_{s} p_{\mathrm{Im}} d s}{\alpha} \sin \frac{\theta}{2}
$$

$$
K_{r}=\frac{\iint_{s} p_{I m} d s}{4 \alpha} \cos \frac{\theta}{2}
$$

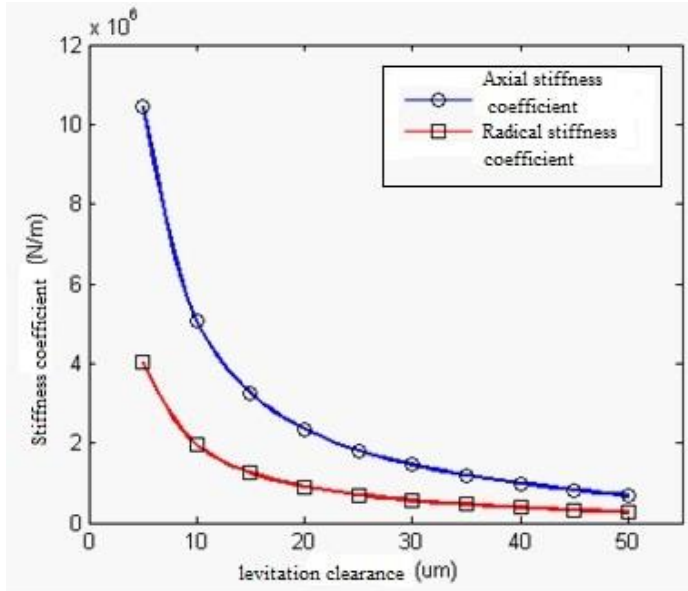

Figure 7. The Relationship between the Stiffness Coefficient and the Levitation Clearance

\section{The Prototype Test}

Before turning on the power supply of the ultrasonic transducer, the end of the rotation shaft and the conical surface of the transducer contact; after turning on the power supply of the ultrasonic transducer, the floated upward movement of the rotation shaft is observed. If we use the gear of measuring on-off in a multimeter to measure the state between the transducer and the rotation shaft, we may find the buzzer does not sound. It means the rotation shaft has been suspended from the surface of the transducer. Moving the rotation shaft along the circumferential direction, it can rotate in the low-speed under the effect of the inertia and show no blocking phenomenon.

Adjusting the position of the transducer at the top with the micrometer head, the axial clearances among two transducers and the motor shaft are gradually decreased, until they reach the minimum values that can ensure the ultrasonic levitation bearing can suspend the motor shaft, and record the scale displayed on the micrometer head. Starting the motor and gradually increasing the speed to observe the levitation state of the motor shaft. In each turn of the motor shaft, the reflective label reflects a photosignal to the sensor of the photoelectric tachometer, which is converted to the electrical signal, recorded by the photoelectric tachometer, and eventually converted to the motor shaft speed. In the process that the motor speed gradually increases, we should reduce the speed gradually by using the frequency converter if the contact friction between the motor shaft and the transducer happens. When the friction noise disappears, record the speed displayed on the photoelectric tachometer. After turning off the power supplies of the motor and the transducer, we can reach the relationship between the maximum speed of the motor and the levitation clearance of the shaft by screwing the micrometer head, increasing the axial clearance between the motor shaft and the transducer gradually, and repeating the above experimental procedures. 


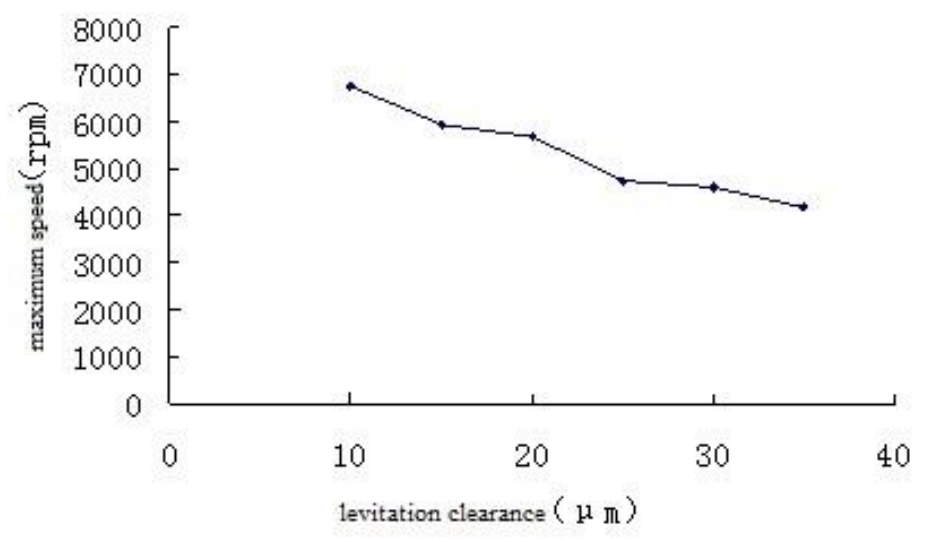

Figure 8. The Relationship between the Maximum Speed and the Levitation Clearance

Being affected by some factors such as the uneven distribution of mass and the stator coil impedance, the motor rotor will show a certain degree of imbalance in the rotation process, which becomes more obvious at a comparatively high speed. At the low speed, the rotor is in a good working condition because of the less effect of the imbalance; however, along with the increase of the rotor speed that is gradually close to the lower order critical speed, the vibration state of the rotor will be intensified by the unbalanced force, and the frictional collision happens.

Figure 8 show that the maximum speed of the motor rotor has a gradually increasing trend along with decreasing the levitation clearance in the support of the ultrasonic levitation. Because there is an antidependence changing relationship between the gas film stiffness of the clearance and the levitation clearance, it means when the bearing clearance is large, the stiffness is small, and the instability of the rotor is easy to happen. By reducing levitation clearance and improving the stiffness of the ultrasonic levitation bearing, the maximum speed of the stable operation for motor rotor can be improved. But the smaller clearance means the higher requirements to the surface finish and the manufacturing accuracy of the bearing.

\section{Conclusion}

In this essay, it has developed an ultrasonic levitation supporting structure to support motor rotor, and the following conclusions have been reached through the theoretical analysis and experiments:

1. By using the ultrasonic levitation support of the structure of piezoelectric transducer with the conical radiation surface, it can form the axial bearing to motor rotor. When the levitation load increases, the clearance decreases.

2. By using the ultrasonic it can achieve the levitation bearing to motor rotor in rotating status. By decreasing the clearance gradually and enhancing the stiffness of the clearance gas film, the maximum speed is improved.

\section{References}

[1] S. Zhang, "The application status and prospect of magnetic suspension technology", Industrial Instrumentation \& Automation, vol. 31, no. 1, (2003).

[2] Y. Zhou, S. Jiagn and L. Zhao, "Progress of research on flywheel energy storasystem", Cryogenics and superconductivity, vol. 31, no. 1, (2003).

[3] Q. Qu, X. Li and J. Zhu, "Ultrathin film lubrication radial bearing simulation tester", Lubrication Engineering, vol. 3, no.18, (1997).

[4] L. Chen, Y. Zhang and Y. Xie, "The Development and application of magnetic bearing", Journal of Northwest Institute of Light Industry, vol. 9, no. 12, (1996). 
[5] H. Yoshiki, K. Yoshikazu and U. Sadayuki, "Noncontact suspending and transporting planar objects by using acoustic levitation", Trans. IEEE of Japan, vol. 117, no.11, (1997).

[6] H. Yoshiki, K. Yoshikazu and U. Sadayuki, "Transporting objects without contact using flexural traveling waves", Journal Acout. Soc. Am., vol. 103, no. 6, (1998).

[7] T. Oiwa and M. Kato, "Squeeze air bearing based on ultrasonic oscillation: Motion compensation using amplitude modulation", Review of Scientific Instruments, vol. 25, no. 75, (2004).

[8] T. A. Stolarski and M.M. Khonsari, "Running Characteristics of Aerodynamic Bearing with Self-Lifting Capability at Low Rotational Speed, Advances in Tribology, vol. 9, no. 12, (2011).

[9] Y. Chang, Z. Yang and B. Wu, "Experimental study on bearing capacity and suspension performance of Ultrasonic Bearing", Piezoelectrics \& Acoustooptics, vol. 27, no. 2, (2004).

[10] Y. Chang, B. Wu and Z. Yang, "The antifriction performance ability and the ultrasonic suspension thrust bearing", Journal of Jilin University, vol. 34, no. 2, (2004).

[11] Y. Chang, T. Peng and J. Kan, "Experiment Study on the Influence on Friction-factor by Ultrasonic Vibration", Piezoelectrics \& Acoustooptics, vol. 37, no. 2, (2004).

[12] T. Peng, S. Yang and Z. Yang, "Experimental study on ultrasonic antifriction behavior", Journal of Jilin University (Engineering and Technology Edition), vol. 36, no. 2, (2009).

[13] P. Zeng, Y. Cao, and G. Cheng, "Analysis of Antifriction Theory of Ultrasonic Bearing", Lubrication Engineering, vol. 182, no. 10, (2006).

[14] Y. Chang, B. Wu and G. Cheng, "Analysis of vibration mode on piezoelectric ceramic actuator for ultrasonic vibration bearing and experimental study of the antifriction", Journal of Harbin Institute of Technology, vol. 68, no. 5, (2006).

[15] B, Wu, Y. Chang and Z. Yang, "Experimental Study and Theoretical Analysis on Anti-friction Capability of Ultrasonic Vibration", China Mechanical Engineering, vol. 15, no. 9, (2004).

[16] T. Peng, Z. Yang and F. Tian, "Investigation on ultrasonic levitative support to high speed axis in fly wheel", Optics and precision engineering, vol. 16, no. 10, (2008).

[17] G. Cheng, P. Zeng and X. Qiu, "Study on ultrasonic vibration antifriction phenomenon", Piezoelectrics \& Acoustooptics, vol. 20, no. 5, (1998)

[18] F. Tian, X. Che and Z. Yang, "Structure design of bidirectional support ultrasonic levitation bearing", Optics and Precision Engineering, vol. 17,no. 4, (2009).

[19] Y. Chang, "Mechanism of Acoustic Levitation and Reducing Fraction Analysis and Basic Experimental Study on the Ultrasonic Bearing", Jilin University, China, (2005).

[20] L. Liu, "Design of Motor Rotor Support Structure and Rotor Dynamics Study Based on Ultrasonic Levitation Technology", Jilin University, China, (2012).

[21] F. Tian, "Research on Supporting Technique about Ultrasonic Near-field acoustic levitation based on Sandwich Piezoelectric Transducer", Jilin University, China, (2012).

[22] E. Matsuo, Y. Koike and K. Nakamura, "Holding characteristics of planar objects suspended by nearfield acoustic levitation", Ultrasonics, vol. 38, no. 4, (2009).

\section{Author}

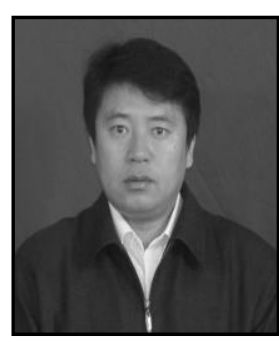

Wang Hong-Chen. Male, he was born in May 1972, Jilin University doctoral student, is mainly engaged in the piezo drive and control technology, small machinery and precision machinery research.

E-mail: whcwanghongchen@126.com Tel: +8615526639260. 